\title{
Inhibition effects of Yuxiao San combined with cisplatin on transplanted tumor growths via upregulation of nm-23 and downregulation of K-ras in Lewis lung cancer mice
}

\author{
MO TANG, SHUMEI WANG, YULIN WEI and JIANTI FU \\ College of Traditional Chinese Medicine, Chongqing Medical University, Chongqing 400016, P.R. China
}

Received August 5, 2017; Accepted November 29, 2017

DOI: $10.3892 / \mathrm{ol} .2018 .9673$

\begin{abstract}
Traditional Chinese medicine applied to cancer therapy has a long history. This study aimed to investigate the effect and underlying mechanism of the traditional Chinese medicine, Yuxiao San, in combination with cisplatin, on Lewis lung cancer mice. Lewis lung cancer mouse models were were established in mice, and were randomly divided into model (normal saline + egg white), cisplatin (cisplatin injection + egg white), traditional Chinese medicine (Yuxiao San + egg white) and combined medication (Yuxiao San + cisplatin injection + egg white) groups. The weight and tumor size of mice was measured every 3 days, and the number of lung surface metastasis nodules were calculated after 14 days. Nucleoside diphosphate kinase (nm-23) and $\mathrm{K}$-ras expression levels in tumor tissues were determined by immunohistochemical methods and reverse transcriptionquantitative polymerase chain reaction. The results suggested that compared with the model group, cisplatin, traditional Chinese medicine and combined medication groups significantly inhibited growth of transplanted tumors, reduced the number of lung metastasis nodules, increased the inhibition rate of lung metastasis $(\mathrm{P}<0.05$ or $\mathrm{P}<0.01)$, significantly increased tumor suppressor gene $\mathrm{nm}-23$ expression and significantly downregulated oncogene K-ras expression. Among all groups, the effect of the combined medication group for inhibiting tumor growth was most favorable $(\mathrm{P}<0.01)$. Compared with the cisplatin group, nm-23 expression in the traditional Chinese medicine group and the combined medication group was significantly increased, and K-ras expression in the traditional Chinese medicine and combined medication groups was significantly reduced $(\mathrm{P}<0.01)$. To conclude, Yuxiao San and cisplatin injection effectively inhibited growth of the transplanted tumor. The underlying mechanism may be
\end{abstract}

Correspondence to: Professor Shumei Wang, College of Traditional Chinese Medicine, Chongqing Medical University, 1 Yi Xueyuan Road, Chongqing 400016, P.R. China

E-mail: tangmoooe@hainan.net

Key words: Yuxiao San, cisplatin, Lewis lung cancer, nucleoside diphosphate kinase, K-ras associate with nm-23 upregulation and K-ras downregulation. Therefore, the Yuxiao San and cisplatin injection may become a potential therapeutic strategy for tumor treatment.

\section{Introduction}

Conventional modern medical treatments of malignant tumors include surgery, radiation therapy, chemotherapy and molecular targeted therapy; however, these methods frequently induce trauma and unwanted side effects, and are poorly tolerated in patients with advanced disease $(1,2)$. Cisplatin is a widely used anti-cancer drug that may induce serious toxicity in the organs and tissues (2). Until now, cisplatin has been extensively used to treat many tumors, including breast, gastric and pancreatic cancer, and cervical carcinoma. A high dose of cisplatin may induce side-effects for patients or in animal models, and the dosage of cisplatin must be reduced. Traditional Chinese medicine applied to cancer therapy has a long history, and has attracted attention (3). In recent years, in the field of cancer therapy, especially for breast cancer and cervical carcinoma, traditional Chinese medicine drugs have alleviated symptoms of tumors $(2,3)$. The traditional Chinese medicine drugs prevented chemotherapy side effects and complications, treatment of cancer pain, severe pleural effusion, severe ascites, cancerous mass, cancer complications and constipation (3). Yang et al (3) reported that traditional Chinese medicines potentially target the tumor cellular proteasome and NF-kB pathway whose activation is dependent on the proteasome activity. Therefore, in order to reduce the side-effects of cisplatin, the present study firstly used traditional Chinese medicine and cisplatin combination chemotherapy to treat tumors in a mouse model of lung cancer, as this particular model may reflect best the therapeutic effects of the aforementioned combination.

Yuxiao San is composed of Curcuma aromatica, mint, rhubarb, pubescent holly root, dandelion and Glauber's salt. In traditional Chinese medicine Yuxiao Sanis believed to treat qi stagnation and blood stasis, eliminate phlegm and remove toxins, to prevent swelling and relieve pain. The present study used the established Lewis lung cancer mouse model (4) and studied the effects and underlying mechanism of Yuxiao San and cisplatin injection in Lewis lung cancer mice. To the best of our knowledge, there are currently no studies published evaluating the effects of Yuxiao San on tumor cell growth or development. The 
objective of the present study is to provide basic data regarding the use of Yuxia San in the clinical treatment of lung cancer.

\section{Materials and methods}

Animals and cell lines. A total of 45 maleC57BL/6 mice weighing 18-22 g, aged from 6 to 8 weeks were purchased from Chongqing Medical University Experimental Animal Center (production license: SCXK-ChongQing-2012-0001). All of the mice were raised in the standard cleaning environment as the following: Temperature $23 \pm 2^{\circ} \mathrm{C}$, humidity $55 \pm 10 \%, 12 \mathrm{~h}$ illumination, noise $\leq 60 \mathrm{~dB}$ and free access to food and water. A Lewis lung cancer cell line, C57BL, was purchased from the West China University Cancer Center (Chengdu, China). The present study was approved by the ethics committee of College of Traditional Chinese Medicine of Chongqing Medical University, Chongqing, China (approval no. 2014052).

Experimental drugs, reagents and instruments. Yuxiao San was purchased from Chongqing Tong Junge Large Pharmacy (Chongqing, China). According to high performance liquid chromatography, Yuxiao San primarily consisted of hexadecenoic acid, $\beta$-sitosterol, burdock oligosaccharide, kikyo saponin, litchi saponin, curcumin and American ginseng total saponin. Cisplatin (cat. no. 2A1A1401002A) was purchased from Qilu Pharmaceutical Co., Ltd. (Hainan, China). Nm-23 and K-ras antibody (cat. nos. bs-0688R and bs-1033R, respectively) were purchased from the Beijing Boosen Biotechnology Co., Ltd (Beijing, China). 3,3'-diaminobenzidene color developing reagent kit (cat. no. K155922C) and Biotin-Streptavidin horseradish peroxidase Detection System (SP test kit; cat. no. 15155A08) were purchased from Beijing Zhongshan Golden Bridge Biotechnology Co., Ltd. (Beijing, China). Formaldehyde (cat. no. 2015050501), glacial acetic acid (cat. no. 2015040201) were purchased from Chengdu Kelong Chemical Co., Ltd. (Chengdu, China). Picric acid (cat. no. P9330) was purchased from the Beijing Solarbio Science \& Technology Co., Ltd. (Beijing, China).

Establishment of the Lewis lung cancer mouse model. Exponentially growing cells were resuspended to $1 \times 10^{7}$ cells $/ \mathrm{ml}$, ready for subcutaneous injection into C57BL/6J mice $(n=5)$. On day 14 , the mice were sacrificed. A cancer cell suspension was prepared by stripping tumor tissue using radioimmunoprecipitation assay (RIPA) buffer at final concentration of $1 \mathrm{M}$ (cat. no. P0013B; Biyotime Biotech. Shanghai, China) and dissolved in HuMEC Basal serum-free medium (cat. no., 12753018, Gibco; Thermo Fisher Scientific, Inc. Waltham, MA, USA). A total of $1 \times 10^{7}$ cells $/ \mathrm{ml}$ (total volume $0.2 \mathrm{ml}$ ) were subcutaneously injected into the dorsal region of the remaining $40 \mathrm{C} 57 \mathrm{BL} / 6 \mathrm{~J}$ mice.

Grouping and administration. After 6 days of growing subcutaneous tumors, the mice were randomly divided into four groups ( $\mathrm{n}=10$ per group): Model group, cisplatin group, traditional Chinese medicine group and combined medication group. In the model group, a sterile gauze was used to administer normal saline and egg white to tumors. In the cisplatin group, cisplatin and egg white was administered to the tumor. In the Yuxiao San group, Yuxiao San and egg white were mixed to form a cream, which was compressed onto the skin to $\sim 2-3 \mathrm{~mm}$ thickness. In the combined medication group, Yuxiao San, cisplatin and albumin were mixed to form a cream, which was compressed onto the skin for $\sim 2-3 \mathrm{~mm}$ thickness. Treatment for every group was performed according to a previously published study (5). The above drugs were administered three times a day, eight $h$ at a time, for 21 days.

Observe the general condition and tumor growth. Before inoculation and after vaccination mice were weighed. General observations were noted, including their hair, diet and activities.

For tumor growth calculations, before and after inoculation, mice were weighed once every three days and the dose of administration was adjusted according to weight change. After 21 days administration, the mice were sacrificed, the tumor was removed and weighed, and then the (a) longest and (b) shortest diameter of mice tumors was determined with a vernier caliper. The volume of tumor was calculated according to the equation: $\mathrm{V}=0.5 \mathrm{ab}^{2}$ (units in $\mathrm{cm}^{3}$ ), and the curve was drawn. The tumor growth inhibition rate was calculated as: (Average weight of tumors in model group-average weight of tumors in treatment group)/average weight of tumors in model group x100\% (6).

Pulmonary surface metastatic nodules. On day 21, the mice were dissected, the pulmonary tissue was removed, and fixed for $24 \mathrm{~h}$ with Bouin's fixative at $4^{\circ} \mathrm{C}$ (picric acid, formaldehyde, glacial acetic acid in a ratio of 75:25:5, respectively), then put them into ethyl alcohol to remove the yellow color. Metastases were observed under a microscope as white nodules. The rate of inhibition on metastasis (\%) was calculated as: (Average metastatic nodules of pulmonary surface of model group-average metastatic nodules of pulmonary surface of treatment group)/average metastatic nodules of pulmonary surface of model group x $100 \%$.

Western blotting assay. The tumor tissue was embedded in paraffin (Sigma-Aldrich; Merck KGaA, Darmstadt, Germany) and cut into $4 \mu \mathrm{m}$ sections, and incubated with RIPA lysis buffer (Sigma-Aldrich; Merck KGaA), and the total proteins were extracted and the concentration was examined using bicinchoninic acid protein assay kit (cat. no. PA115; Tiangen Biotech Co. Ltd., Beijing, China). Equal quantities of protein $(2 \mu \mathrm{g})$ were separated using a 10\% gel and SDS-PAGE and electro transferred onto polyvinylidene difluoride membranes (EMD Millipore, Billerica, MA, USA). Subsequently, the membranes were blocked with 5\% non-fat milk for $2 \mathrm{~h}$ at $4^{\circ} \mathrm{C}$. The membranes were incubated with a rabbit anti-mouse Nm23 polyclonal antibody (1:2,000; cat. no. ab154547; Abcam, Cambridge, UK), a rabbit anti-mouse K-ras monoclonal antibody (1:3,000; cat. no. ab199557; Abcam) and a rabbit anti-mouse GAPDH polyclonal antibody $(1: 2,000$; cat. no. ab9485; Abcam) for $2 \mathrm{~h}$ at room temperature. The membranes were then incubated with horseradish peroxidase-conjugated goat anti-rabbit $\operatorname{IgG}(1: 2,000$, cat. no. sc-2030; Santa Cruz Biotechnology, Inc., Dallas, TX, USA) at $37^{\circ} \mathrm{C}$ for $1 \mathrm{~h}$. Finally, the western blot bands were visualized by using an enhanced chemiluminescent kit (Thermo Fisher Scientific, Inc.). Finally, the bands were scanned and analyzed by using a UVP gel image scanning system GDS8000 software (UVP, Sacramento, CA, USA). 
Reverse transcription-quantitative polymerase chain reaction $(R T-q P C R)$. RNA was extracted from tissues using TRIzol (Tiangen Biotech Co. Ltd.). The concentration and purity of RNA was measured by determining the optical density by using a spectrophotometer (Nanodrop Lite; Thermo Fisher Scientific, Inc.). cDNA was synthesized by using a Rever Tre Ace-a-reverse transcription kit (cat. no. FKS-101, Toyobo Life Science, Osaka, Japan). The primers were: nm-23: Forward, 5'-CGGCAGTGA TTCAGTGGAGAGT-3' and reverse, 5'-CTGGTTTCTCCG CGTCTACTCATAC-3'; K-ras: Forward, 5'-CTGGGGAGG GCTTTCTTTGTG-3' and reverse, 5'-CTCCTGAGCCTGTTT CGTGTCTA-3'; $\beta$-actin: Forward, 5'-ACCCCGTGCTGCTGA CCGAG-3' and reverse, 5'-TCCCGGCCAGCCAGGTCCA-3'. The RT reaction conditions were: $42^{\circ} \mathrm{C}$ for $10 \mathrm{~min}, 30^{\circ} \mathrm{C}$ for $20 \mathrm{~min}, 99^{\circ} \mathrm{C}$ for $5 \mathrm{~min}$ and $4^{\circ} \mathrm{C}$ for $5 \mathrm{~min}$. The PCR reaction conditions were: $94^{\circ} \mathrm{C}$ for $5 \mathrm{~min}, 94^{\circ} \mathrm{C}$ for $30 \mathrm{sec}, 57^{\circ} \mathrm{C}$ for $30 \mathrm{sec}$ and $72^{\circ} \mathrm{C}$ for $30 \mathrm{sec}$ for a total of 40 cycles and termination at $72^{\circ} \mathrm{C}$ for $10 \mathrm{~min}$. After the reaction, the ABI StepOne Plus PCR system was used to analyze and calculate the cycle threshold value of the target gene. The relative mRNA expression of targeting genes was normalized to the $\beta$-actin gene by using the comparative threshold cycle $\left(2^{-\Delta \Delta \mathrm{Cq}}\right)$ method (7).

Statistical analysis. Data in the present study were analyzed using SPSS software version 19.0 (IBM Corp., Armonk, NY, USA). Data was presented as the mean \pm standard deviation. All data were obtained from at least three independent experiments. Tukey's post hoc test was used following a one-way analysis of variance between the two groups. $\mathrm{P}<0.05$ was considered to indicate a statistically significant difference.

\section{Results}

General observations. A total of 40 mice were inoculated with Lewis lung carcinoma cells and all developed tumors. Mice experienced symptoms of lassitude, asthenia, somnolence, lethargy and withered hair. Compared with the model group, these symptoms were reduced in the Traditional Chinese medicine group and combined medication group. However, the cisplatin group not only exacerbated the above symptoms, but also suffered side-effects of chemotherapy, such as decreased appetite, loss of weight and loss of hair (data not shown).

Comparison of tumor growth in each group. The weight of tumors in the cisplatin, Yuxiao San group and the combination group were lower than that of the model group $(\mathrm{P}<0.01$; Table I). The weight of tumor in the Yuxiao San group and combination group were lower than the cisplatin group $(\mathrm{P}<0.01$; Table I). The combination group had a lower tumor weight than the cisplatin group and Yuxiao San group $(\mathrm{P}<0.01$; Table I).

The volume of tumor in the cisplatin group, Yuxiao San group and the combination group were lower than that of the model group $(\mathrm{P}<0.01$; Table $\mathrm{I})$. The volume of tumor in the Yuxiao San group and combination group were lower than that in the cisplatin group $(\mathrm{P}<0.01$; Table I). The combination group had a lower volume of tumor than the cisplatin group and Yuxiao San group $(\mathrm{P}<0.01$; Table I). The inhibition rate of tumor growth in the combination group was $55.88 \%$ and was higher than the cisplatin group $(33.82 \%)$ and Yuxiao San group $(43.14 \%)(\mathrm{P}<0.01$; Table I).
Comparison of pulmonary surface metastatic nodules in each group. The rate of inhibition on tumor metastasis in the cisplatin group, Yuxiao San group and the combination group (35.90, 57.38 and $68.85 \%$, respectively) was significantly increased compared with that of the model group (24.60\%) $(\mathrm{P}<0.01$; Table II). The combination group also inhibited pulmonary surface metastatic nodules to a greater extent than the other groups (Table II).

Observation for $K$-ras and nm-23 expression in tumor tissue by using immunohistochemistry. K-ras and nm-23 were detected via immunohistochemical staining (Fig. 1). The mean absorbance of K-ras expression in the model group, cisplatin group, Yuxiao San group and combination group were $1.29 \pm 0.01,1.02 \pm 0.24,0.87 \pm 0.17$ and $0.612 \pm 0.02$, respectively (Table III). Compared with the model group, expression of K-ras and nm-23 in the other groups were significantly decreased $(\mathrm{P}<0.01)$. Compared with the model group, the cisplatin group and the Yuxiao San group, the combination group exhibited the most significantly decreased expression levels of K-ras and nm-23 ( $\mathrm{P}<0.01$; Table III). Compared with the cisplatin group, the Yuxiao San group and combination group exhibited significantly decreased expression of the two proteins $(\mathrm{P}<0.01)$. Compared with the Yuxiao San group, the combination group exhibited the most significantly decreased expression levels. For the decreased expression of K-ras and $\mathrm{nm}-23$, the combination of cisplatin and Yuxiao San was superior to single drug treatment $(\mathrm{P}<0.01$; Table III).

The average absorbance values of $\mathrm{nm}-23$ in the model group, cisplatin group, Yuxiao San group and combination group were $0.58 \pm 0.02,0.65 \pm 0.01,0.89 \pm 0.01$ and $1.08 \pm 0.02$, respectively (Table III). Compared with the model group, the other groups exhibited significantly greater expression levels of nm-23 ( $\mathrm{P}<0.01$; Table III). Compared with the model group, cisplatin group and Yuxiao San group, the combination group exhibitedthe most significantly increased expression of nm-23 $(\mathrm{P}<0.01$; Table III). Compared with the cisplatin group, the Yuxiao San group and combination group exhibited significantly increased nm-23 expression levels ( $\mathrm{P}<0.01$; Table III). Compared with Yuxiao San group, the combination group demonstrated the most significantly increased nm-23 expression levels $(\mathrm{P}<0.01$; Table III). For the decreased expression of K-ras and nm-23, the combination of cisplatin and Yuxiao San is superior to single drug treatment $(\mathrm{P}<0.01$; Table III).

Detection of $K$-ras and nm-23 in tumor tissue by western blotting. K-ras expression levels were examined by using western blotting (Fig. 2A). The results suggested that K-ras protein expression levels in the cisplatin group, Yuxiao San group and cisplatin and Yuxiao San combination group were significantly decreased compared with the model group $(\mathrm{P}<0.01$; Fig. 2B). In addition, K-ras expression levels in the Yuxiao San group and the cisplatin and Yuxiao San combination group were also decreased compared with the cisplatin group $(\mathrm{P}<0.01$; Fig. $2 \mathrm{~B})$.

Protein expression levels of $\mathrm{nm}-23$ in the cisplatin group, Yuxiao San group, and cisplatin and Yuxiao San combination group were significantly increased compared with the model group $(\mathrm{P}<0.01$; Fig. $2 \mathrm{~B})$. Protein expression levels of nm-23 in the Yuxiao San group and cisplatin and Yuxiao 
Table I. Effect of Yuxiao San on Lewis lung cancer tumor growth and progression in C57BL/6 mice (mean \pm standard deviation, $\mathrm{n}=10$ per group).

\begin{tabular}{lllc}
\hline Group & Weight $(\mathrm{g})$ & Volume $\left(\mathrm{cm}^{3}\right)$ & Inhibition rate $(\%)$ \\
\hline Model group & $2.04 \pm 0.36$ & $2.88 \pm 0.63$ & - \\
Cisplatin group & $1.35 \pm 0.16^{\mathrm{a}}$ & $1.72 \pm 0.25^{\mathrm{a}}$ & 33.82 \\
Yuxiao San group & $1.16 \pm 0.11$ & $1.27 \pm 0.23^{\mathrm{a}, \mathrm{b}}$ & 43.14 \\
Yuxiao San + cisplatin group & $0.94 \pm 0.17^{\mathrm{a}-\mathrm{c}}$ & $0.90 \pm 0.13^{\mathrm{a}-\mathrm{c}}$ & 55.88 \\
\hline
\end{tabular}

${ }^{\mathrm{a}} \mathrm{P}<0.01$ vs. model group; ${ }^{\mathrm{b}} \mathrm{P}<0.05$ vs. cisplatin group; ${ }^{\mathrm{c}} \mathrm{P}<0.01$ vs. Yuxiao San group.

Table II. Effect of Yuxiao San on pulmonary surface metastatic nodules of Lewis lung carcinoma in C57BL/6 mice (mean \pm standard deviation, $\mathrm{n}=10$ per group).

\begin{tabular}{lcc}
\hline Group & $\begin{array}{c}\text { Pulmonary } \\
\text { metastatic } \\
\text { nodules (n) }\end{array}$ & $\begin{array}{c}\text { Metastatic } \\
\text { inhibition } \\
\text { rate (\%) }\end{array}$ \\
\hline Model group & $6.10 \pm 1.59$ & 24.60 \\
Cisplatin group & $3.91 \pm 1.20^{\mathrm{a}}$ & 35.90 \\
Yuxiao San group & $2.60 \pm 1.43^{\mathrm{a}, \mathrm{b}}$ & 57.38 \\
Yuxiao San + cisplatin group & $1.90 \pm 1.19^{\mathrm{c}}$ & 68.85 \\
\hline
\end{tabular}

${ }^{\mathrm{a}} \mathrm{P}<0.01$ vs. model group; ${ }^{\mathrm{b}} \mathrm{P}<0.05$ and ${ }^{\mathrm{c}} \mathrm{P}<0.01$ vs. cisplatin group.

San combination group were also increased compared to the cisplatin group ( $\mathrm{P}<0.01$; Fig. 2B).

Detection of K-ras and nm-23 in tumor tissue by RT-qPCR. K-ras mRNA expression levels were examined by using RT-qPCR (Fig. 3). The results suggested that K-ras mRNA expression levels in the cisplatin group, Yuxiao San group and cisplatin and Yuxiao San combination group were significantly decreased compared with the model group $(\mathrm{P}<0.01)$. K-ras mRNA expression levels in the Yuxiao San group and cisplatin and Yuxiao San combination group were also decreased compared with the cisplatin group $(\mathrm{P}<0.01)$.

In addition, $\mathrm{nm}-23 \mathrm{mRNA}$ expression levels in the cisplatin group, Yuxiao San group and cisplatin and Yuxiao San combination group were significantly increased compared with the model group ( $\mathrm{P}<0.01$; Fig. 3). nm-23 mRNA expression levels in the Yuxiao San group and cisplatin and Yuxiao San combination group were increased compared with the cisplatin group $(\mathrm{P}<0.01)$.

\section{Discussion}

The traditional Chinese Medicine holds the view that tumors are associated with the depression and stress indicated in patients. Lung cancer also belongs to a kind of pulmonary disease, according to a Chinese medical theory, indicating an abscess in the lung (8). Yuxiao San powder is a common application for tumors and is composed of Curcuma aromatica, mint, rhubarb, pubescent holly root, dandelion and Glauber's salt $(9,10)$. Modern medical research has established that the effective constituent of Curcuma aromatica (8), mint (9) and dandelion (10) work against tumor growth and induce cell apoptosis.

The K-ras gene causes cancer when mutated. K-ras genes make proteins, which are involved in cell signaling, growth and death (apoptosis). The Ras oncogene is well known to be involved in cancer development, including H-RAS, K-RAS and N-RAS, which encode a family of $21 \mathrm{kDa}$ guanosine triphosphate-binding proteins called $\mathrm{p} 21$.

In physiologic conditions, these proteins may be activated by binding with guanosine triphosphate (GTP) and initiate cell proliferation via the Ras-dependent kinase cascade. Subsequently, GTP is hydrolyzed to guanosine diphosphate by its intrinsic GTPase activity and Ras proteins return to an inactive state. However, in tumors, when a point mutation occurs in these genes, which often results in loss of its intrinsic GTPase activity, Ras proteins may acquire transforming potential, leading to continuous activation of Ras signaling (11).

K-ras gene mutations occur frequently in non-small cell lung cancer (NSCLC), mainly in adenocarcinoma, and rarely in squamous cell carcinoma (12). Approximately $80 \%$ of K-ras mutations in NSCLC involve codon 12, others are located in codons 13 or 61 (13). Evidence from animal model studies of NSCLC demonstrated that K-ras mutations enhance cellular proliferation and induce malignant transformation, and their continuous activation serves a key role in tumor development and maintenance. The carcinogenesis of K-ras mutations was also demonstrated in human NSCLC (14).

Targeted knockdown of K-ras in NSCLC cell lines has provided critical preclinical confirmation of the role of this driver in tumorigenesis, resulting in the suppression of tumor growth and sensitization to inhibitors of other signaling pathways (15). However, in practical terms, K-ras itself is an intractable target for the development of therapeutics (16), and considerable effort has thus focused on inhibition of its downstream effectors to perturb persistent activation of oncogenic signaling pathways. The canonical RAF/MEK/ERK kinase cascade is the primary mitogenic pathway stimulated by K-ras under both physiologic and pathologic conditions $(17,18)$.

The nm-23 gene was first discovered as a cancer metastasis suppressor in murine melanoma cell lines. As many as five nm-23 genes in human have been identified, including $\mathrm{nm}-23-\mathrm{h} 1$ to $\mathrm{nm}-23-\mathrm{h} 5$. Protein $\mathrm{nm}-23$ gene products are known as abnormal wing disc, which is found in Drosophila (19). Factor 1 (inhibitor of differentiation) and c-myc purine-binding transcription factor (PUF) are 
Table III. Comparison of groups of the mean absorbance of K-ras and nm23 (mean \pm standard deviation, $\mathrm{n}=10$ per group).

\begin{tabular}{lccc}
\hline Group & Dose $(\mathrm{g} / \mathrm{kg})$ & K-ras & $\mathrm{nm}-23$ \\
\hline Model group & - & $1.29 \pm 0.01$ & $0.58 \pm 0.02$ \\
Cisplatin group & 0.02 & $1.02 \pm 0.24^{\mathrm{a}}$ & $0.65 \pm 0.01^{\mathrm{a}}$ \\
Yuxiao San group & 10 & $0.87 \pm 0.17^{\mathrm{a}, \mathrm{b}}$ & $0.89 \pm 0.01^{\mathrm{a}, \mathrm{b}}$ \\
Yuxiao San + cisplatin group & $10 / 0.02$ & $0.61 \pm 0.02^{\mathrm{a}-\mathrm{c}}$ & $1.08 \pm 0.02^{\mathrm{a}-\mathrm{c}}$
\end{tabular}

${ }^{\mathrm{a}} \mathrm{P}<0.01$ vs. model group; ${ }^{\mathrm{b}} \mathrm{P}<0.01$ vs. cisplatin group; ${ }^{\mathrm{c}} \mathrm{P}<0.01$ vs. Yuxiao San group.

A

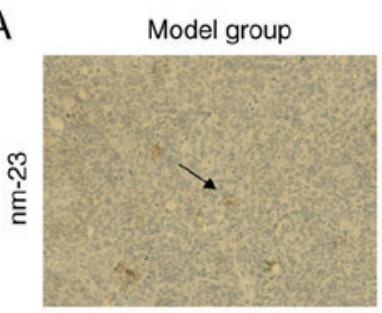

B

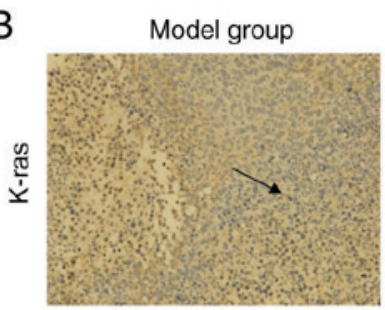

Cisplatin group

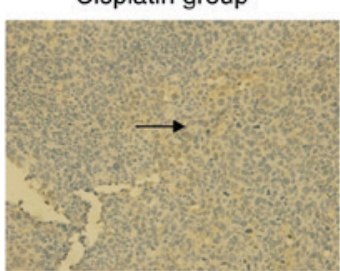

Cisplatin group

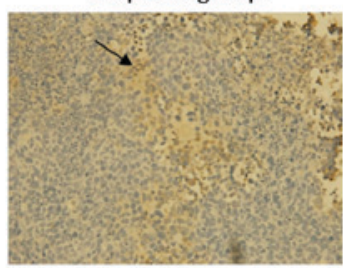

Yuxiao San group

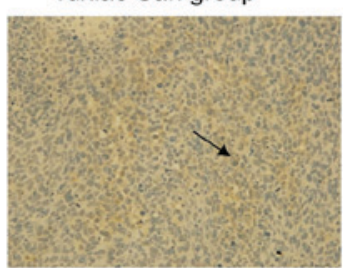

Yuxiao San group

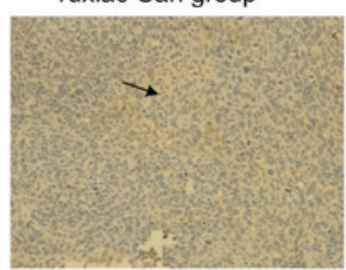

Yuxiao Sant Cisplatin group

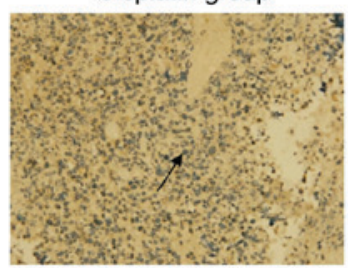

Yuxiao San+ Cisplatin group

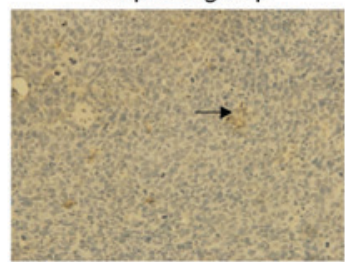

Figure 1. Immunohistochemical staining of (A) nm-23 and (B) K-ras expression. Magnification, x400. nm-23, nucleoside diphosphate kinase.

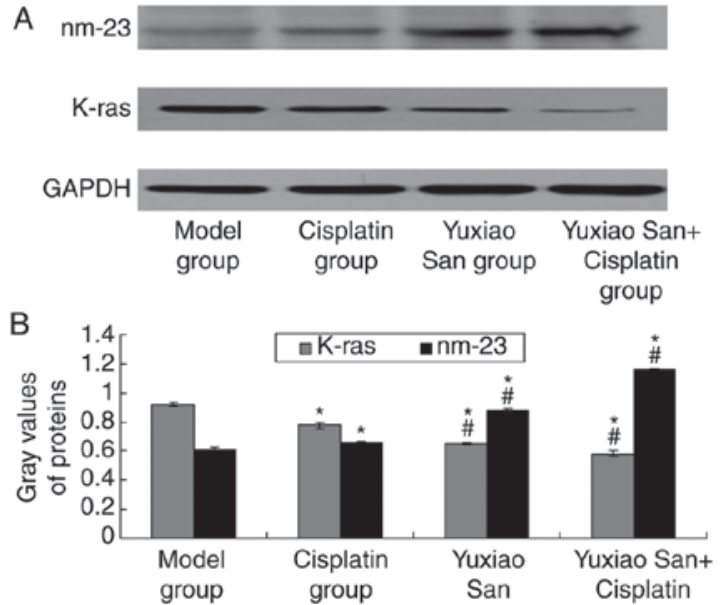

Figure 2. Expression of K-ras and nm-23 by western blotting. (A) Western blotting and (B) quantification and statistical analysis. ${ }^{*} \mathrm{P}<0.01$ vs. model group and ${ }^{\#} \mathrm{P}<0.01$ vs. cisplatin group. $\mathrm{nm}-23$, nucleoside diphosphate kinase.

the transcription factors that regulate c-myc. Factor 1 and PUF include a large family of proteins that have activity for nucleoside diphosphate kinase (NDPK). NDPK is found in almost all cells and serves to catalyze the phosphorylation of 5 'triphosphate nucleoside to 5'diphosphate nucleoside, through an intermediary mechanism involving high-energy oxidative phosphorylation enzymes $(19,20)$.

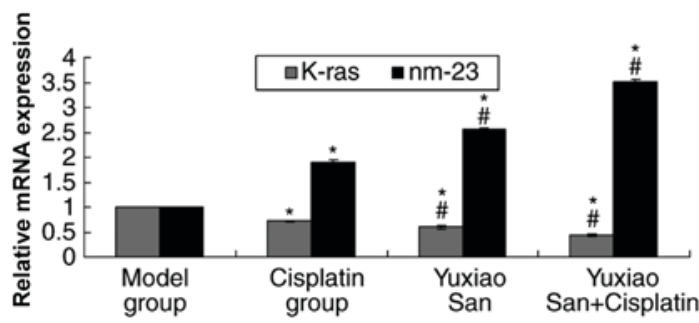

Figure 3. mRNA expression levels of K-ras and nm-23 using reverse transcription-quantitative polymerase chain reaction. * $\mathrm{P}<0.01$ vs. model group and ${ }^{\#} \mathrm{P}<0.01$ vs. cisplatin group. $\mathrm{nm}-23$, nucleoside diphosphate kinase.

NDPK serves a function in signal transduction from the cell membrane into the cell nucleus via a $\mathrm{G}$ protein activation pathway and $\mathrm{p} 21$, and serves a role in cell division and maintenance of cell shape (21). Protein nm-23 gene products have implications in the proliferation and differentiation of cells, and also serves a role in cancer (22). The nm-23 protein is distributed to the cytosol, mitochondria, plasma membrane and nucleus (23). The nm-23 gene family is highly conserved among a wide variety of eukaryotic species $(24,25)$. The identification of nm23 as a tumor suppressor gene indicated the existence of genes that specifically regulate metastasis $(26,27)$.

The function of nm23 in human cancer is currently unconfirmed (22,28-31), and certain reports have indicated there is a link between high expression of the nm-23 gene and its 
protein product with low metastatic potential of cancer (28). $\mathrm{Nm}-23$ is known to be associated with early onset of familial breast and ovarian cancer (28). Furthermore, an inverse association between nm-23 expression and metastasis was also observed in various types of cancer (22). Although the mechanism by which nm-23 regulates metastasis is not fully understood, experimental data have shown that nm23 serves an important role in the regulation of metastasis in a number of human cancer types (29). It was also reported that nm-23 expression was a significant factor for predicting a favorable prognosis, suggesting the anti-metastatic potential of nm-23 in NSCLC $(30,31)$. The expression of nm 23 in normal lung tissue was much higher than in cancer tissues, and was associated with staging and lymphatic metastasis, and this may suggest that the absence of nm23 expression may serve an important role in the incidence of NSCLC.

The present study determined the effects of Yuxiao San on Lewis lung cancer in mice. This study was conducted in vivo and in vitro, and there were four groups, including the model group, cisplatin group, Yuxiao San group and the combination group (Yuxiao San and cisplatin). The Lewis lung cancer model in C57BL/6 mice was established and mice were treated with Yuxiao San and cisplatin, before determining any side effects of treatment. Comparisons between tumor weight, tumor volume and tumor inhibition rate were made, and the methods of immunohistochemistry, western blotting and RT-qPCR were used to detect K-ras and nm 23 gene expression levels in tumor tissue.

Results of the present study revealed that Yuxiao San may reduce tumor weight and tumor volume. Yuxiao San may also decrease K-ras gene expression levels and increase $\mathrm{nm}-23$ gene expression levels. It was found that Yuxiao San downregulated the expression of K-ras, and upregulating expression of nm-23 in Lewis lung cancer mice tissues. The expression levels of nm-23 in normal lung tissue was much higher compared with that in cancer tissues. Combining cisplatin and Yuxiao San was better than the use of single drug treatment, as compared with the model group, the tumor volume, weight and lung metastases of tissues treated with Yuxiao San and cisplatin group in combination were decreased. In addition, compared with the model group, the expression levels of K-ras via immunohistochemical staining and RT-qPCR were decreased. Expression levels of nm-23 in tumors were increased via immunohistochemistry staining, western blotting and RT-qPCR.

Although this study revealed some notable results, there were a number of limitations. Firstly, the sample sizes were relatively small. In a subsequent study, a large sample size should be utilized. Furthermore, the down-stream factors of nm-23 and K-ras have not been clarified. The mechanism for the effect of Yuxiao San following the changes of nm-23 and K-ras should be investigated. Additionally, the effects of Yuxiao San have not been examined in combination with the other chemotherapy drugs, except for cisplatin. In a follow-up study, the combined effects of Yuxiao San with other chemotherapy drugs should be assessed.

To conclude, Yuxiao San and cisplatin injection by external application may effectively inhibit growth of transplanted tumors in mice. The underlying mechanism may be associated with nm-23 upregulation and K-ras downregulation.

\section{Acknowledgements}

Not applicable.

\section{Funding}

The present study was supported by National Clinical Research Base of traditional Chinese medicine Construction in 2015 (second special research projects; grant no. JDZX2015073) and Project of Chongqing Education Bureau (grant no. yjg143075).

\section{Availability of data and materials}

All data generated or analyzed during this study are included in this published article.

\section{Authors' contributions}

MT, SW, YW performed the tests and experiments. YW analyzed the data. MT and JF wrote the manuscript and were the major contributors to the design of the study. All authors read and approved the final manuscript.

\section{Ethics approval and consent to participate}

The present study was performed following the guidelines of the Care and Use of Laboratory Animals of National Institute of Health (NIH) and was approved by the Ethics Committee of Chongqing Medical University (Chongqing, China).

\section{Patient consent for publication}

Not applicable.

\section{Competing interests}

The authors declare that they have no competing interests.

\section{References}

1. Ye L, Jia Y, Ji KE, Sanders AJ, Xue K, Ji J, Mason MD and Jiang WG: Traditional Chinese medicine in the prevention and treatment of cancer and cancer metastasis. Oncol Lett 10: $1240-1250,2015$

2. Li F and Zhang W: Role of traditional Chinese medicine and its chemical components in anti-tumor metastasis. J Cancer Res Ther 10 (Suppl 1): S20-S26, 2014.

3. Yang H, Liu J and Dou QP: Targeting tumor proteasome with traditional Chinese medicine. Curr Drug Discov Technol 7: 46-53, 2010.

4. Takahashi Y, Izumi Y, Matsutani N, Dejima H, Nakayama T, Okamura R, Uehara $\mathrm{H}$ and Kawamura M: Optimized magnitude of cryosurgery facilitating anti-tumor immunoreaction in a mouse model of Lewis lung cancer. Cancer Immunol Immunother 65: 973-982, 2016.

5. Wu C, Wang Y, Xia Y, He S, Wang Z, Chen Y, Shu Y and Jiang J: Wilms' tumor 1 enhances Cisplatin-resistance of advanced NSCLC. FEBS Lett 588: 4566-4572, 2014.

6. Li Y, Gu JF, Zou X, Wu J, Zhang MH, Jiang J, Qin D, Zhou JY, Liu BX, Zhu YT, et al: The anti-lung cancer activities of steroidal saponins of P. polyphylla Smith var. chinensis (Franch.) Hara through enhanced immunostimulation in experimental Lewis tumor-bearing C57BL/6 mice and induction of apoptosis in the A549 cell line. Molecules 18: 12916-12936, 2013.

7. Livak KJ and Schmittgen TD: Analysis of relative gene expression data using real-time quantitative PCR and the 2(-Delta Delta C(T)) method. Methods 25: 402-408, 2001. 
8. Zhang F, Xu L, Qu X, Zhao M, Jin B, Kang J, Liu Y and Hu X Synergistic antitumor effect of $\beta$-elemene and etoposide is mediated via induction of cell apoptosis and cell cycle arrest in non-small cell lung carcinoma cells. Mol Med Rep 4: 1189-1193, 2011.

9. Journigan VB and Zaveri NT: TRPM8 ion channel ligands for new therapeutic applications and as probes to study menthol pharmacology. Life Sci 92: 425-437, 2013.

10. Loh CH, Inbaraj BS, Liu MH and Chen BH: Determination of chlorophylls in Taraxacum formosanum by high-performance liquid chromatography-diode array detection-mass spectrometry and preparation by column chromatography. J Agric Food Chem 60: 6108-6115, 2012

11. Ma Y, Guo FC, Wang W, Shi HS, Li D and Wang YS: K-ras gene mutation as a predictor of cancer cell responsiveness to metformin. Mol Med Rep 8: 763-768, 2013.

12. Rodenhuis S, van de Wetering ML, Mooi WJ, Evers SG, van Zandwijk N and Bos JL: Mutational activation of the K-ras oncogene. A possible pathogenetic factor in adenocarcinoma of the lung. N Engl J Med 317: 929-935, 1987.

13. Weiss GJ, Ganeshan B, Miles KA, Campbell DH, Cheung PY, Frank $\mathrm{S}$ and Korn RL: Noninvasive image texture analysis differentiates K-ras mutation from pan-wildtype NSCLC and is prognostic. PLoS One 9: e100244, 2014.

14. Piva S, Ganzinelli M, Garassino MC, Caiola E, Farina G, Broggini $\mathrm{M}$ and Marabese $\mathrm{M}$ : Across the universe of K-RAS mutations in non-small-cell-lung cancer. Curr Pharm Des 20: 3933-3943, 2014

15. Sunaga N, Shames DS, Girard L, Peyton M, Larsen JE, Imai H, Soh J, Sato M, Yanagitani N, Kaira K, et al: Knockdown of oncogenic KRAS in non-small cell lung cancers suppresses tumor growth and sensitizes tumor cells to targeted therapy. Mol Cancer Ther 10: 336-346, 2011.

16. Young A,Lyons J,Miller AL, Phan VT, Alarcón IR and McCormick F: Ras signaling and therapies. Adv Cancer Res 102: 1-17, 2009.

17. Dalpa E, Gourvas V, Soulitzis N and Spandidos DA: K-Ras, H-Ras, $\mathrm{N}-\mathrm{R}$ as and B-Raf mutation and expression analysis in Wilms tumors: Association with tumor growth. Med Oncol 34: 6, 2017.

18. Lee JT Jr, Steelman LS and McCubrey JA: Modulation of Raf/MEK/ERK kinase activity does not affect the chemoresistance profile of advanced prostate cancer cells. Int J Oncol 26 : $1637-1644,2005$

19. Conery AR, Sever S and Harlow E: Nucleoside diphosphate kinase Nm23-H1 regulates chromosomal stability by activating the GTPase dynamin during cytokinesis. Proc Natl Acad Sci USA 107: 15461-15466, 2010.
20. Gong L, Wu Z, Guo L, Li L, Zhao R, Zhu D and Zhou Q: Metastasis suppressor Nm23-H1 inhibits STAT3 signaling via a negative feedback mechanism. Biochem Biophys Res Commun 434: 541-546, 2013

21. Fujita Y, Fujiwara K, Zenitani S and Yamashita T: Acetylation of NDPK-D regulates its subcellular localization and cell survival. PLoS One 10: e0139616, 2015.

22. Qu L, Liang L, Su J and Yang Z: Inhibitory effect of upregulated DR-nm23 expression on invasion and metastasis in colorectal cancer. Eur J Cancer Prev 22: 512-522, 2013.

23. Radović S, Dorić M, Hukić A, Babić M, Kuskunović S and Spahović N: Immunohistochemical expression and significance of NM23 suppressor protein in primary gastric adenocarcinoma. Bosn J Basic Med Sci 13: 72-77, 2013.

24. Schlattner U, Tokarska-Schlattner M, Epand RM, Boissan M, Lacombe ML, Klein-Seetharaman $J$ and Kagan VE: Mitochondrial NM23-H4/NDPK-D: A bifunctional nanoswitch for bioenergetics and lipid signaling. Naunyn Schmiedebergs Arch Pharmacol 388: 271-278, 2015.

25. Durán E, Cárdenas JM, Reina MA and Arriazu R: Loss of Nm23 is associated with a more favorable tumor microenvironment in patients with breast cancer. Histol Histopathol 30: 345-352, 2015.

26. Yokdang N, Nordmeier S, Speirs K, Burkin HR and Buxton IL: Blockade of extracellular NM23 or its endothelial target slows breast cancer growth and metastasis. Integr Cancer Sci Ther 2: 192-200, 2015.

27. Niitsu N: The association of $\mathrm{nm} 23-\mathrm{H} 1$ expression with a poor prognosis in patients with peripheral T-cell lymphoma, not otherwise specified. J Clin Exp Hematop 54: 171-177, 2014.

28. Zhang X, Fu LJ, Liu XQ, Hu ZY, Jiang Y, Gao RF, Feng Q, Lan X, Geng YQ, Chen XM, et al: nm23 regulates decidualization through the PI3K-Akt-mTOR signaling pathways in mice and humans. Hum Reprod 31: 2339-2351, 2016.

29. Fiore LS, Ganguly SS, Sledziona J, Cibull ML, Wang C, Richards DL, Neltner JM, Beach C, McCorkle JR, Kaetzel DM and Plattner R: c-Abl and Arg induce cathepsin-mediated lysosomal degradation of the NM23-H1 metastasis suppressor in invasive cancer. Oncogene 33: 4508-4520, 2014.

30. You J, Chang R, Liu B, Zu L and Zhou Q: Nm23-H1 was involved in regulation of KAI1 expression in high-metastatic lung cancer cells L9981. J Thorac Dis 8: 1217-1226, 2016.

31. Wu Y, Li Y, Zhao X, Dong D, Tang C, Li E and Geng Q: Combined detection of the expression of Nm23-H1 and p53 is correlated with survival rates of patients with stage II and III colorectal cancer. Oncol Lett 13: 129-136, 2017. 